\title{
Untypisch lokalisierte Pseudo-Acanthosis nigricans bei einem 11-Jährigen mit Colitis ulcerosa
}

\section{Kowalzick \\ H. Ziegler}

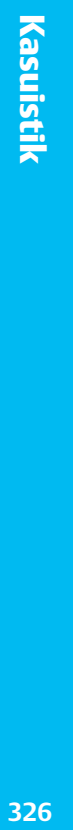

\section{Einleitung}

Von einer Pseudo-Acanthosis nigricans wird gesprochen, wenn Hauterscheinungen einer Acanthosis nigricans bestehen, ohne dass eine unterliegende maligne Erkrankung detektiert werden kann. Zu den Auslösern einer Pseudo-Acanthosis nigricans gehören Adipositas und Stoffwechselstörungen. Typischerweise sind die Hautveränderungen in den Intertrigines oder großen Gelenkbeugen lokalisiert. Wir berichten hier über den Fall eines Knaben mit untypisch lokalisierten Hauterscheinungen einer PseudoAcanthosis nigricans, bei dem zudem eine langjährige Colitis ulcerosa bestand, die mit Kortikosteroiden und Azathioprin behandelt wurde.

\author{
with Colitis Ulcerosa
}

\section{Abstract}

We report the case of an 11-year-old boy with benign acanthosis nigricans localized at the dorsal side of both hands. Because of a colitis ulcerosa from which he was suffering since his third year a longstanding treatment with systemic glucocorticosteroids was administered. The present skin eruptions occurred after an additional immunosuppressive therapy with azathioprine. Following the termination of azathioprine and further tapering of the steroids combined with an intensive topical treatment with tretionin and urea, a marked improvement of the skin lesions resulted.

\section{Kasuistik}

Uns wurde ein nicht-übergewichtiger 11-jähriger Knabe mit symmetrisch lokalisierten Hautveränderungen im Bereich beider Handrücken, genauer im Bereich beider Tabatièren sowie der Knöchelpolster der Mittelfinger-Grundgelenke, vorgestellt. Die relativ scharf begrenzten, schmutzig grau-braun pigmentierten, hyperkeratotischen, etwas schwielenartigen, die Hautfältelung deutlich betonenden Plaques (Abb.1 und 2) wären vor etwa 8 Wochen plötzlich entstanden und hätten sich dann nicht mehr verändert. Juckreiz würde nicht bestehen. Das sonstige Integument, insbesondere die Intertrigines und Gelenkbeugen fand sich unauffällig. Eine besondere oder neue mechanische Belastung an den Handrücken wurde anamnestisch ausgeschlossen. In der Familienanamnese fand sich kein Hinweis auf heriditäre Verhornungsstörungen, speziell Palmoplantarkeratosen. 

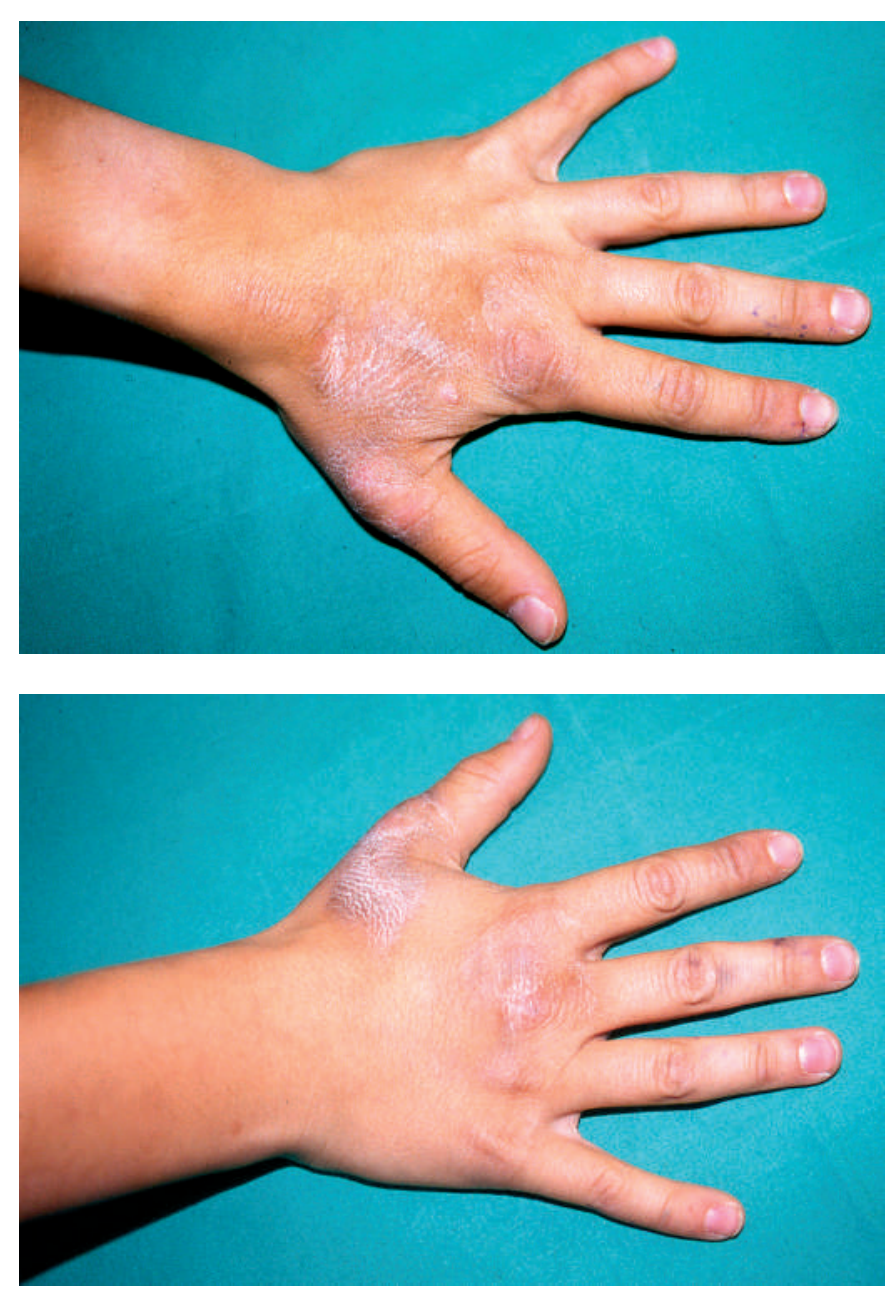

Abb. 1 und 2 Pseudo-Acanthosis nigricans an den Dorsalseiten (Tabatière-Region und Knöchelpolster der Mittelfinger-Grundgelenke) beider Handrücken bei 11-jahrigem Jungen mit Colitis ulcerosa und kombinierter immunsuppressiver Therapie mit Steroiden und Azathioprin.

Atopische Erkrankungen wurden gleichfalls nicht angegeben. Auf genaues Befragen gab die Mutter des Patienten an, dass der Patient seit seinem dritten Lebensjahr an Darmbeschwerden leidet, die Diagnose einer Colitis ulcerosa wurde vor 7 Jahren gestellt. Zu diesem Zeitpunkt erhielt der Patient erstmals Azathioprin in Kombination mit Steroiden. Wegen eines neuen Schubes der entzündlichen Darmerkrankung erhielt der Patient seit einem Jahr erneut Azathioprin in einer Dosis von zunächst $25 \mathrm{mg}$, später $50 \mathrm{mg}$ pro Tag. Außerdem wurde systemisch Prednisolon in einer täglichen Dosis von $20 \mathrm{mg}$ gegeben. Die Hautveränderungen im Sinne der Acanthosis nigricans traten am Ende der einjährigen Azathioprin-Gabe auf. Ein Anhalt für eine maligne Erkrankung besteht bei regelmäßigen pädiatrischen Kontrollen nicht, so dass von einer Pseudo-Acanthosis nigricans ausgegangen werden kann. Da unter der Kombination von Azathioprin und Kortikosteroiden in bis zu $10 \%$ der Fälle das Auftreten einer Pseudo-Acanthosis nigricans berichtet wurde [7], rieten wir dazu, soweit es die Darmerkrankung zuließe, auf die weitere Gabe von Azathioprin zu verzichten, und nach Möglichkeit auch die Steroiddosis zu reduzieren. Darüber hinaus verordneten wir eine intensive Therapie mit einem tretionin- und harnstoffhaltigen Externum (Carbamid + VAS Creme Widmer ${ }^{\circledR}$ ). Hierunter und nach Reduktion der täglichen systemischen Steroiddosis auf
$10 \mathrm{mg}$ bei Verzicht auf erneute Gabe von Azathioprin, kam es binnen zwei Monaten zu einer drastischen Besserung der Hauterscheinungen der Pseudo-Acanthosis nigricans.

\section{Diskussion}

Üblicherweise ist die Acanthosis nigricans, bzw. bei Ausschluss einer unterliegenden malignen Erkrankung, die Pseudo-Acanthosis nigricans in den Intertrigines, insbesondere den Axillen, aber auch Leisten und den großen Gelenkbeugen, insbesondere im Nacken aber auch am Hals, den Ellenbeugen und Kniekehlen lokalisiert. Die Lokalisation im vorliegenden Fall an den Handrücken ist als eher untypisch anzusehen. Vereinzelt wird zwar ein Mitbefall von Palmae und Plantae („Tripe“ palms = „Pansen“Handflächen) [6,8] oder auch der Handrücken [9] beschrieben, jedoch erscheint der isolierte Befall der Handrücken als ungewöhnlich. Möglicherweise kann aber zumindest die TabatiéreRegion gleichfalls als intertriginöse Lokalisation im weiteren Sinne aufgefasst werden.

Bei Kindern und Adoleszenten, insbesondere Übergewichtigen mit Insulinresistenz oder Typ 2 Diabetes mellitus ist eine Pseudo-Acanthosis nigricans nicht selten [1,2,5]. Bei Patienten mit immunsuppressiver Therapie mit Kortikoiden und Azathioprin nach Nierentransplantation wird eine Pseudo-Acanthosis nigricans in 0,5 bis $10 \%$ der Fälle beschrieben $[4,7]$. So wurde auch der Fall eines 12-jährigen Nierentransplantierten mit Befall der Ellenbeugen, des Halses und des Nabels beschrieben, der allerdings mit Steroiden in Kombination mit Cyclosporin A therapiert wurde, und bei dem die Hauterscheinungen bereits 3 Monate nach Beginn der immunsuppressiven Therapie begannen [10]. Eine besondere Häufung von Pseudo-Acanthosis nigricans bei chronisch entzündlichen Darmerkrankung wurde dagegen noch nicht beschrieben. Lediglich Einzelfälle, darunter einer bei einem Patienten, der außer an einer Colitis ulcerosa zugleich an systemischem Lupus erythematodes mit Hepatitis litt und langfristig mit Steroiden therapiert wurde, wurden beschrieben [3].

Offen muss im vorliegenden Fall bleiben, ob die Steroide, das Azathioprin oder die Kombination als auslösend für die Hauterscheinungen anzusehen sind. Für eine wesentliche Beteiligung von Azathioprin spräche, dass sich die Symptome nach Elimination dieser Substanz besserten. Allerdings wurde die Steroiddosis gleichzeitig gesenkt, so dass auch dies, neben der intensiven Lokaltherapie, einen Einfluss gehabt haben kann. Schließlich wäre zu diskutieren, ob die relativ hohe langzeitige Dosierung von Steroiden eine Insulinresistenz oder einen subklinischen Diabetes mellitus 2 zur Folge gehabt haben kann, so dass hierdurch eine metabolisch verursachte Form der Pseudo-Acanthosis nigricans entstanden sein könnte. 
Literatur

${ }^{1}$ Bolding J, Wratchford T, Perkins K, Ogershok P. Prevalence of obesity, acanthosis nigricans and hyperinsulinemia in an adolescent clinic. WV Med J 2005; 101: 112-115

${ }^{2}$ Brockow K, Steinkraus V, Rinninger F, Abeck D, Ring J. Acanthosis nigricans: a marker for hyperinsulinemia. Pediatr Dermatol 1995; 12: $323-326$

${ }^{3}$ Brown J, Winkelmann RK. Acanthosis nigricans: a study of 90 cases. Medicine (Baltimore) 1968; 47: 3-51

4 l'Eplattenier JL, Binswanger U, Ott F, Largiadère F. Dermatologische Komplikationen bei immunsupprimierten Patienten nach Nierentransplantation. Schweiz Med Wschr 1980; 110: 1307-1313

${ }^{5}$ Fu JF, Liang L, Don GP, Jiang YJ, Zou CC. Obese children with benign acanthosis nigricans and insulin resistence: analysis of 19 cases. Zhonghua Er Ke Za Zhi 2004; 42: 917-919
${ }^{6}$ Hazen PG, Carney JF, Walker AE, Stewart JJ. Acanthosis nigricans presenting as hyperkeratosis of the palma and soles. J Am Acad Dermatol 1979; 1 : 541 - 544

${ }^{7}$ Koranda FC, Dehmel EM, Kahn G, Penn I. Cutaneous complications in immunosuppressed renal homograft recipients. JAMA 1974; 229 : $419-424$

${ }^{8}$ Möhrenschlager M, Vocks E, Wessner DB, Nahrig J, Ring J. Tripe palms and malignant acanthosis nigricans: cutaneous signs of imminent metastasis in bladder cancer? J Urol 2001; 165: 1629-1630

${ }^{9}$ Nair PS, Moorthy PK, Suprakasan S, Jayapalan S, Sarin M. Malignant acanthosis nigricans with liver secondaries from an occult primary adenocarcinoma of gastrointestinal tract. Indian J Dermatol Venereol Leprol 2005; 71: 197 - 198

10 v. Schnakenburg C, Enke B, Jürgens K, Offner G. Pseudo-Acanthosis nigricans bei einem 12 Jahre alten Jungen nach Nierentransplantation. Klin Pädiatr 2001; 213: 288 - 289

\section{Buchbesprechung}

\section{Kompendium Dermatologie}

Thomas Brinkmeier

Heidelberg: Springer, 2006. 1042 S., Kart. 36,95€, ISBN 3-540-25720-9

Bilder sind die Welt der Dermatologie. Aber ein abbildungsfreies Nachschlagewerk? Als Kompendium - in seiner Konzeption ein neuer Publikationstyp in der Dermatologie - versteht sich das vorliegende Buch nicht als Konkurrenz zu Lehrbüchern und Lexika der Dermatologie. Der praktische Wert eines Kompendiums lebt von Kürze, Vollständigkeit und Aktualität. Das vorliegende Werk reflektiert den umfassenden Kenntnisstand seines Autors zur dermatologischen Facharztprüfung.

Nach kurzgefasster Benutzeranleitung und einem umfangreichen Verzeichnis der zahlreich verwendeten Abkürzungen folgen 12 Seiten dermatologischer Differenzialdiagnosen nach klinischer Effloreszenzenlehre. Dabei kommen auch Feinheiten zur Sprache wie z.B. die Differenzialdiagnostik der WimpernHypertrichose (außer bei HIV auch z. B. bei SLE oder maligner Grunderkrankung). Der anschließende Lexikonteil umfaßt rund 1000 Seiten alphabetisch gelisteter Krankheitsbilder, daneben auch diagnostischer Verfahren (z.B. minimale Erythemdosis) oder klassischer Therapeutika (z. B. Glukokortikoide). Tabellarische Übersichten erleichtern den Zugriff etwa auf die gültige TNM-Klassifikation und aktuelle Nachsorgeempfehlungen des malignen Melanoms.

Die klare Gliederung zu den jeweiligen Stichworten wird durch ein Raster von Abkürzungen am Zeilenanfang vorgegeben: Beispielhaft von „Historisch (Histr) und „Synonyme (Syn)“ über ein „Cave (CV)“ zu „Ätiologie (Ät)“, „Pathogenese (Pg)“ und z. B. „Histologie (Hi)“ bis zu „Therapie (Th)“ und aktueller „Literatur (Lit)“. Lediglich das Kürzel „GS“ (wohl „Goldstandard“?) hat sich der Leser selbst zusammenzureimen.
Auf dem neuesten Stand befinden sich die Ausführungen zur Therapie, etwa zum „GS Penicillin“ bei Lues: Clemizol-Penicillin derzeit nicht verfügbar! Für Tacrolimus findet man ein differenziertes Spektrum an Indikationen, z. B. bei Lichen ruber mucosae oder bei Vitiligo. Nahrungsmittelallergien (11 Seiten!) sind ebenso vertreten wie Details zur Hyposensibilisierung gegen Aeroallergene oder Insektengifte.

Man findet wenige Stichworte, bei denen Verbesserungen wünschenswert wären. Hierzu zählt z. B. der extramammäre Morbus Paget, für den ein fakultativ assoziiertes Malignom des benachbarten Urogenitaltrakts nicht genannt wird. Die Empfehlung, den Rubinlaser alternativ zur Exzision bei erworbenen makulösen Pigmentnaevi einzusetzen, sollte zumindest relativiert werden. Redundanz ließe sich z. B. durch Zusammenfassung der aufeinanderfolgenden Stichworte „Epitheloides Sarkom“ und „Epitheloidsarkom" vermeiden.

Als Adressaten werden neben dem dermatologischen Fachpublikum auch Medizinstudenten und Kollegen anderer Disziplinen wie Allgemeinmedizin, Pädiatrie oder Gynäkologie genannt. Die hohe Verdichtung der Information dürfte aber für einen leichten Zugang dermatologische Vorbildung erfordern. Dann aber wird jeder Nutzer diese Fundgrube praxisnaher Informationen wertschätzen, und dies zu einem moderaten Preis.

Zusammenfassend eine beeindruckende Fleißarbeit, die sich aber nicht mit dem Status quo erschöpfen wird. Um seine heutige Gültigkeit auch für die Zukunft zu sichern, wird das Kompendium laufender Aktualisierungen bedürfen. Besonders für die Therapieangaben gilt es Schritt zu halten mit der sprichwörtlich abnehmenden Halbwertszeit des medizinischen Wissenszuwachses.

D. und B. Krahl, Heidelberg 